\title{
Metodología utilizada para estimar la mortalidad atribuible a alcohol en España, 2001-2017
}

\section{Methodology used to estimate alcohol- attributable mortality in Spain, 2001-2017}

\author{
Marta Donat*,**, Luis Sordo**,***, Juan Miguel Guerras*******, Julieta Politi****, José \\ Pulido******, Gregorio Barrio ***. \\ * Escuela Nacional de Sanidad. Instituto de Salud Carlos III, Madrid. \\ ** Centro de Investigación Biomédica en Red de Epidemiología y Salud Pública (CIBERESP). \\ *** Departamento de Salud Pública y Materno-infantil, Facultad de Medicina, Universidad Complutense \\ de Madrid. \\ **** Centro Nacional de Epidemiología. Instituto de Salud Carlos III, Madrid.
}

\section{Resumen}

El objetivo es describir y discutir los métodos y asunciones para estimar la mortalidad atribuible a alcohol en España en 2001-2017. Se estimó el $\mathrm{n}^{\circ}$ medio anual de muertes atribuibles a alcohol (MAAs) basándose en 19 grupos de causas de muerte relacionadas con alcohol (18 parcialmente atribuibles y uno directamente atribuible), y 20 fracciones atribuibles poblacionales al alcohol (FAPs) para cada grupo de causas, resultantes de combinar sexo, 5 grupos de edad, y los períodos 2001-2009 y 2010-2017. Las muertes por causa se obtuvieron del Instituto Nacional de Estadística. Para las causas parcialmente atribuibles se calcularon FAPs específicas para España, usando la fórmula de Levin con datos de exposición al alcohol procedentes de encuestas de salud y estadísticas de ventas, y riesgos relativos procedentes de metanálisis internacionales. Se consideraron las prevalencias anuales de exbebedores y de siete niveles de consumo diario de alcohol. Se corrigió la subestimación del consumo medio diario autoinformado con respecto a las estadísticas de venta, multiplicando por un factor de 1,58-3,18, dependiendo del año-calendario. Se calcularon tasas de MAA y porcentajes de la mortalidad general atribuibles a alcohol estandarizados por edad, según sexo, grupo de edad, periodo-calendario, tipo de bebedor y comunidad autónoma. Se realizaron análisis de sensibilidad observando cómo cambiaban las estimaciones de MAA al hacerlo algunas opciones metodológicas, como el criterio de exbebedor o la introducción de un período de latencia.

Palabras clave: alcohol, mortalidad atribuible, España, metodología.

\begin{abstract}
The objective is to describe and discuss methods and assumptions to estimate the mortality attributable to alcohol in Spain in 2001-2017. The annual mean number of deaths attributable to alcohol (DAAs) was estimated based on 19 groups of alcohol-related causes of death (18 partially attributable and one directly attributable), and 20 alcohol population-attributable fractions (PAFs), resulting from combining sex, 5 age groups, and the periods 2001-2009 and 2010-2017, for each cause group. Deaths from causes were obtained from the Spanish National Institute of Statistics. For partially attributable causes, Spain-specific PAFs were calculated using the Levin formula with alcohol exposure data from health surveys and sales statistics, and relative risks from international meta-analyses. Annual prevalences of ex-drinkers and seven levels of daily alcohol consumption were considered. The underestimation of self-reported daily average consumption with respect to the sales statistics was corrected by multiplying by a factor of 1.58-3.18, depending on the calendar year. DAA rates standardized by age and standardized proportions of general mortality attributable to alcohol, according to sex, age group, calendar period, type of drinker and autonomous community were calculated. Sensitivity analyses were performed to assess how the DAA estimates changed when changing some methodological options, such as the ex-drinker criterion or the introduction of a latency period. Key words: alcohol, attributable mortality, Spain, methodology.
\end{abstract}


$\mathrm{E}$ 1 consumo de alcohol es uno de los principales factores de riesgo prevenibles de morbimortalidad y discapacidad en muchos países (Global Burden of Disease [GBD], 2018a; Institute for Health Metrics and Evaluation [IHME], 2020). Las personas consumidoras de alcohol, especialmente bebedores de alto riesgo o personas con trastorno por uso de alcohol, tienen un riesgo de muerte mucho más elevado que la población general (Roerecke y Rehm, 2013). Sin embargo, el consumo de alcohol empieza a aumentar el riesgo de muerte mucho antes de que se alcancen niveles de consumo considerados de alto riesgo, o antes de que surja un trastorno por uso de alcohol (Stockwell et al., 2016).

La estimación de las muertes atribuibles a alcohol (MAA) permite obtener indicadores sintéticos esenciales para comparar el impacto del uso de esta sustancia entre territorios, períodos y subgrupos, determinar la necesidad de intervenciones de salud pública, evaluarlas y asignar recursos (World Health Organization [WHO], 2009). La Organización Mundial de la Salud define las MAA como la suma algebraica de las muertes causadas y prevenidas por el consumo de alcohol que no se habrían producido en un escenario contrafactual sin consumo histórico de esta sustancia. En teoría, deberían estimarse comparando el riesgo real de muerte con el riesgo hipotético en un escenario contrafactual sin consumo histórico de alcohol (WHO, 2018a).

Sin embargo, las estimaciones necesitan información procedente de múltiples fuentes, que a menudo muestran lagunas importantes, por lo que distintos autores suelen realizar elecciones metodológicas y asunciones no siempre coincidentes, y/o acudir a datos no siempre extrapolables sobre consumo de alcohol procedentes de otros momentos o territorios. Entre otros aspectos, se pueden producir elecciones metodológicas referidas a las causas de muerte relacionadas con alcohol incluidas, los estudios o metanálisis que proporcionan las funciones de riesgo que relacionan la cantidad de alcohol consumida con la mortalidad por cada causa, la consideración o no del riesgo de MAA en exbebedores o del asociado al consumo intensivo episódico (atracones de alcohol) y sus definiciones, el $\mathrm{n}^{\circ} \mathrm{de}$ subgrupos para el que se obtienen las fracciones atribuibles poblacionales a alcohol (FAP) utilizadas en los cálculos, la consideración o no de un período de latencia entre consumo de alcohol y la muerte, y la corrección o no de la subestimación del consumo promedio autoinformado en las encuestas con respecto a fuentes de mayor validez.

Por las razones mencionadas a veces circulan estimaciones bastante dispares para un mismo país, lo que introduce mucha incertidumbre a la hora de utilizar los resultados en la toma de decisiones. España no es ajena a esta situación, encontrándose estimaciones del número anual de MAA en la población de 15 años y más, realizadas por autores o instituciones nacionales o internacionales, que van desde 8558 en 1999-2004 (Fierro, Ochoa, Yánez, Valderrama y
Álvarez, 2008) a 37000 en 2016 (GBD, 2018b), y la proporción de la mortalidad general atribuible a alcohol desde $2,1 \%$ a $9,0 \%$, respectivamente. Sin embargo, la estimación más baja se basó solo parcialmente en datos empíricos de consumo obtenidos de encuestas españolas, y esos datos no se corrigieron por la subestimación del consumo promedio autoinformado en las encuestas poblacionales (Fierro et al., 2008). En cuanto a las estimaciones realizadas por instituciones extranjeras o internacionales (GBD, 2018a; IHME, 2020; WHO, 2020), estas emplean fuentes de datos internacionales, por lo que sus datos se basan en la distribución poblacional del consumo promedio y de los patrones de consumo de alcohol no específicos de España. Además, algunos detalles metodológicos son opacos o difíciles de conocer, lo que imposibilita aplicarlas para obtener resultados distintos a los publicados, referidos, por ejemplo, a distintos subgrupos de interés.

Parece, pues, necesario que un país como España disponga de procedimientos detallados que permitan la estimación de las MAA basándose en datos sobre la distribución poblacional del consumo de alcohol recogidos directamente de la población española. Ello permitirá hacer estimaciones rutinarias rápidas del riesgo de MAA, incluyendo series sobre su evolución temporal y comparaciones interterritoriales (por ejemplo, entre comunidades autónomas) y entre subgrupos sociodemográficos. En este sentido, y más allá de las estimaciones globales que puedan existir, muchos países, sobre todo los de tradición anglosajona, disponen de metodologías propias para hacer estas estimaciones rutinarias.

El objetivo de este artículo es describir y discutir una metodología propia para estimar la mortalidad atribuible a alcohol en España, que se aplicará en un artículo posterior para obtener estimaciones durante el período 2001-2017.

\section{Descripción de la metodología}

\section{Enfoque metodológico general}

Se consideran MAA las muertes causadas y prevenidas por el consumo de alcohol que no se habrían producido en un escenario contrafactual sin consumo histórico de esa sustancia. Para estimar el $n^{\circ}$ total de MAA se utiliza el enfoque de causa específica. Para ello, se seleccionan una serie de causas o grupos de causas que se consideran relacionadas con el consumo de alcohol, se estiman las muertes atribuibles a alcohol para cada una de esas causas (MAAc) y se suman los resultados de todas las seleccionadas.

$$
\mathrm{MAA}=\Sigma \mathrm{MAA}_{\mathrm{c}}
$$

El $n^{\circ}$ de MAA se estima multiplicando el $n^{\circ}$ de muertes por esa causa $\left(\mathrm{N}_{\mathrm{c}}\right)$, extraído de las estadísticas de mortalidad, por su FAP correspondiente $\left(\mathrm{FAP}_{\mathrm{c}}\right)$, que es la proporción de muertes por esa causa atribuibles a la exposición al alcohol y que podrían evitarse si la población dejara completamente de consumir esta sustancia, y expresa la contribución proporcional del consumo de alcohol a la mor- 
talidad poblacional por dicha causa. Dicha FAP se obtiene mediante un algoritmo que incorpora los riesgos relativos (RR) en relación a los abstemios y las prevalencias poblacionales de distintas categorías de consumo de alcohol.

$$
\mathrm{MAA}_{\mathrm{c}}=\left(\mathrm{N}_{\mathrm{c}}\right)\left(\mathrm{FAP}_{\mathrm{c}}\right)
$$

Para cada causa el proceso se estratifica para diferentes subgrupos poblacionales de sexo, edad y período-calendario, con el objetivo de alcanzar un equilibrio que permita obtener FAP $_{c}$ suficientemente específicas que den una mayor validez a los resultados mientras se mantiene un nivel de precisión razonable. En concreto, para cada causa se obtienen $\mathrm{FAP}_{\mathrm{C}}$ para los 20 subgrupos resultantes de combinar ambos sexos, cinco grupos de edad (15-24, 25-44, $45-64,65-74$ y $\geq 75$ ) y dos periodos calendarios (2001-2009 y 2010-2017). Esto significa que se obtienen estimaciones independientes para cada uno de los 20 subgrupos y se suman con posterioridad. La estimación se refiere a la población de 15 años y más.

Así pues, el número total de muertes atribuibles a alcohol es la suma de las muertes atribuidas a diferentes causas. Por lo que el proceso consiste en: (1) identificar y cuantificar las diferentes causas de muerte relacionadas, (2) establecer para cada causa y subgrupo poblacional una fracción atribuible y, (3) sumar los resultados en cifras absolutas y tasas.

\section{Causas de muerte relacionadas con el alcohol}

Se seleccionan dos tipos de causas de muerte relacionadas con el alcohol: 1) Causas directamente o completa- mente atribuibles a alcohol, en las que se considera que el alcohol es siempre causa necesaria, como el trastorno por uso de alcohol, y 2) Otras causas de muerte relacionadas con el alcohol, en las que el alcohol es un factor contribuyente pero no el único, que comprende 18 grupos, por ejemplo el cáncer de esófago. Las selección de causas se basa en las revisiones y metanálisis más recientes centrados en la valoración del riesgo de desarrollar o morir por determinadas enfermedades asociadas al consumo de alcohol (Corrao, Bagnardi, Zambon y Arico, 1999; Rehm et al., 2017; Samokhvalov, Irving y Rehm, 2010; Sherk, Stockwell, Rehm, Dorocicz y Shield, 2017), y en las selecciones efectuadas en las estimaciones de otros países (Connor, Kydd, Rehm y Shield, 2013; Jones y Bellis, 2013; Marmet, Rehm, Gmel, Frick y Gmel, 2014; Rey, Boniol y Jougla, 2010). En la Tabla 1 se recogen los códigos de la Clasificación Internacional de Enfermedades, décima edición (CIE-10), correspondientes a los 19 grupos de causas seleccionados. Para evitar duplicidades, se excluyen del listado de códigos del grupo de causas directamente atribuibles a alcohol aquellos códigos correspondientes a causas que forman parte de categorías más amplias ya incluidas en algún grupo de causas parcialmente atribuibles. Es el caso de hepatopatía alcohólica (K70), ya incluido en cirrosis/hepatopatía crónica; envenenamiento involuntario por alcohol (X45) o autoenvenenamiento intencional por alcohol (X65), incluidos como causas externas, entre otros (Tabla 1).

Tabla 1. Grupos de causas de muerte seleccionados para estimar la mortalidad atribuible a alcohol en España 2001-2017.

\begin{tabular}{|c|c|}
\hline \multirow[t]{2}{*}{ Grupos de causas } & Códigos CIE-10 $0^{3}$ \\
\hline & Causas parcialmente atribuibles a alcohol \\
\hline 1. Tuberculosis & A15-A19, B90, K67.3, P37.0 \\
\hline 2. Infección respiratoria baja/neumonía & A48.1, A70, J09-J15.8, J16, J20-J21, P23.0-P23.4 \\
\hline 3. Cáncer de boca y faringe ${ }^{1}$ & $\mathrm{C} 00-\mathrm{C} 13$ \\
\hline 4. Cáncer de esófago ${ }^{1}$ & $\mathrm{C} 15$ \\
\hline 5. Cáncer colorrectal ${ }^{1}$ & $\mathrm{C} 18-\mathrm{C} 21$ \\
\hline 6. Cáncer hepático ${ }^{1}$ & $\mathrm{C} 22$ \\
\hline 7. Cáncer de laringe ${ }^{1}$ & C32 \\
\hline 8. Cáncer de mama (mujeres) ${ }^{1}$ & $\mathrm{C} 50$ \\
\hline 9. Diabetes mellitus & E10.0-E10.1, E10.3-E11.1, E11.3-E12.1, E12.3-E13.1, E13.3-E14.1, E14.3-E14.9, P70.0-P70.2, R73 \\
\hline 10. Epilepsia & $\mathrm{G} 40, \mathrm{G} 41$ \\
\hline 11. Cardiopatía hipertensiva & 111 \\
\hline 12. Cardiopatía isquémica & $120-125$ \\
\hline 13. Fibrilación/aleteo auricular & 148 \\
\hline 14. Ictus isquémico & $G 45,163,167.2-167.3,167.5-167.6,169.3$ \\
\hline 15. Ictus no isquémico & $160-162,169.0-169.2,167.0-167.1$ \\
\hline 16. Cirrosis/hepatopatía crónica & $\mathrm{B} 18, \mathrm{I} 85,198.2, \mathrm{~K} 70, \mathrm{~K} 71.3-\mathrm{K} 71.5, \mathrm{~K} 71.7, \mathrm{~K} 72.1-\mathrm{K} 74.6, \mathrm{~K} 75.8-\mathrm{K} 76.0, \mathrm{~K} 76.6-\mathrm{K} 76.7, \mathrm{~K} 76.9$ \\
\hline 17. Pancreatitis & K85-K86 \\
\hline 18. Causa externa & V01-Y98 \\
\hline 19. Causas directamente atribuibles a alcohol ${ }^{2}$ & $\mathrm{E} 24.4, \mathrm{~F} 10, \mathrm{G} 31.2, \mathrm{G} 62.1, \mathrm{G} 72.1, \mathrm{I42.6}, \mathrm{K} 29.2,035.4, \mathrm{R} 78.0$ \\
\hline
\end{tabular}

Nota. 1 No se incluyen códigos D (carcinoma in situ, tumor benigno y tumor de comportamiento incierto o desconocido), porque a menudo no se dispone de un cuarto dígito. 2 No se consideran las causas de muerte directamente atribuibles a alcohol que forman parte de categorías más amplias incluidas en grupos de causas parcialmente atribuibles como hepatopatía alcohólica (K70), envenenamiento involuntario por alcohol (X45), autoenvenenamiento intencional por alcohol (X65), intoxicación por alcohol con intención indeterminada (Y15) y evidencia de participación en el alcohol (Y9o-Y91). 3 CIE-10: Clasificación Estadística Internacional de Enfermedades y Problemas Relacionados con la Salud. Décima Revisión. 
No se incluyen como causas parcialmente atribuibles un amplio grupo de enfermedades o problemas de salud probablemente relacionados con el alcohol, como ciertos cánceres (por ejemplo, de estómago y páncreas) por considerarse insuficiente la evidencia para establecer la causalidad del alcohol o para cuantificar la relación de riesgo (Rehm et al., 2017; WHO, 2018b). Tampoco se incluye la enfermedad por VIH a pesar de que se hace en algunos países (Gmel, Shield y Rehm, 2011; Marmet, Rehm y Gmel, 2016).

El número de muertes por las causas seleccionadas procede del registro de mortalidad del Instituto Nacional de Estadística [INE] (INE, 2020).

\section{Fracciones atribuibles poblacionales al alcohol}

La fracción atribuible poblacional al alcohol para una causa o grupo de causas de muerte $\left(\mathrm{FAP}_{\mathrm{c}}\right.$ ) expresa la contribución relativa del consumo de alcohol a la mortalidad total por esa causa. De esta manera, para las causas de muerte directamente atribuibles a alcohol se aplicó una fracción atribuible de uno $\left(\mathrm{FAP}_{c}=1\right)$, mientras que para las causas parcialmente atribuibles a alcohol se calculan FAP específicas para España. Para el cálculo se utilizó la fórmula de Levin para datos categóricos, que combina datos de prevalencia de distintos niveles de exposición al alcohol con los riesgos relativos de mortalidad por una causa dada (Rehm, Klotsche y Patra, 2007), a la que se ha añadido un término para incluir la fracción que corresponde a exbebedores. La fórmula se muestra a continuación:

$$
\text { FAPc }=\frac{\left[\mathrm{P}_{\mathrm{exb}}\left(\mathrm{RR}_{\mathrm{exb}}-1\right)+\sum_{\mathrm{i}=1}^{\mathrm{n}}\left(\mathrm{P}_{\mathrm{i}}\left(\mathrm{RR}_{\mathrm{i}}-1\right)\right)\right]}{1+\left[\mathrm{P}_{\mathrm{exb}}\left(\mathrm{RR}_{\mathrm{exb}}-1\right)+\sum_{\mathrm{i}=1}^{\mathrm{n}}\left(\mathrm{P}_{\mathrm{i}}\left(\mathrm{RR}_{\mathrm{i}}-1\right)\right)\right]}
$$

En esta fórmula $\mathrm{P}_{\mathrm{exb}}$ es la prevalencia de exbebedores, $\mathrm{P}_{\mathrm{i}}$ la prevalencia para la categoría $i$ de cantidad media de alcohol consumida diariamente durante los últimos 12 meses en la población, $\mathrm{RR}_{\mathrm{exb}}$ el riesgo relativo de exbebedores frente a personas no expuestas (abstemias), y $\mathrm{RR}_{\mathrm{i}}$ el riesgo relativo de las expuestas frente a abstemias para esa causa de muerte y categoría $i$ de cantidad de alcohol consumida diariamente. El número de categorías $i$ utilizadas en los cálculos fue de 7 , correspondientes a los intervalos $\leq 19,20-39$, 40-49, 50-59, 60-79, 80-99 y $\geq 100$ gramos de alcohol puro/ día. Para cada uno de los 18 grupos de causas de muerte parcialmente atribuibles a alcohol se calculan 20 FAPs, resultantes de combinar sexo, 5 grupos de edad, y los períodos 2001-2009 y 2010-2017. Se optó por calcular las FAP para dos períodos plurianuales en vez de hacerlo para cada año-calendario, que aparentemente hubiese proporcionado estimaciones de mayor validez, porque las prevalencias anuales de consumo que se incluyen en la fórmula de cálculo hubiesen estado sometidas a una mayor variabilidad por efecto del azar, sobre todo teniendo en cuenta que hay que estimarlas simultáneamente por estrato de edad, sexo y cantidad de alcohol consumida. Las FAPs calculadas se muestran en la Tabla 2.

La fórmula citada permite segmentar el numerador para estudiar distintos grupos de bebedores (Sherk et al., 2017), por ejemplo, exbebedores y dos grupos de bebedores actuales, con alto riesgo y medio-bajo riesgo, definidos por determinados valores $i$. Se consideran exbebedores aquellas personas que no han consumido bebidas alcohólicas durante el último año, pero las han consumido al menos 12 veces algún año de su vida, siguiendo la definición de la Encuesta Nacional de Salud de Estados Unidos (Villarroel, Clarke y Schoenborn, 2016). Son abstemias las personas que han bebido menos de 12 veces cualquier año de su vida y no han bebido durante el último año. Los bebedores actuales de alto riesgo son hombres (o mujeres) que han consumido una media $\geq 60$ (o 40) g de alcohol puro/día durante el último año, lo que corresponde a 6 (ó 4) unidades de bebida estándar (UBE). Este criterio se adopta de acuerdo con algunos trabajos previos (Marmet et al., 2014; Rehm et al., 2017; Rehm, Rehm, Shield, Gmel y Gual, 2013; Rehm, Shield, Rehm, Gmel y Frick, 2012), incluyendo a bebedores de alto y muy alto riesgo de la clasificación de la OMS (WHO, 2000) y de la Agencia Europea de Medicamentos [EMA] (EMA, 2010; Mann, Aubin y Witkiewitz, 2017). Las demás personas que han consumido alcohol durante el último año se consideran bebedores actuales de medio-bajo riesgo. A la hora de distribuir la MAA según tipo de bebedor, se asume que todas las muertes directamente atribuibles a alcohol $(\mathrm{FAPc}=1)$ se producen en bebedores actuales de alto riesgo y que no se producen muertes por causas externas atribuibles a alcohol en exbebedores regulares.

A continuación se detalla el significado y forma de obtención de los distintos parámetros de la fórmula de Levin utilizada para calcular las FAPs.

\section{Riesgos relativos para distintas categorías de exposi- ción poblacional al alcohol}

El riesgo relativo (RR) es una medida de la fuerza de la asociación entre la exposición al alcohol y la muerte por una determinada causa en un grupo, lugar y tiempo dados en comparación con otro grupo. Los $\mathrm{RR}_{i}$ empleados para el cálculo de las FAPc pueden consultarse en la Tabla 3. Tienen como referencia o escenario contrafactual $(\mathrm{RR}=1)$ a las personas abstemias y para los bebedores actuales se calculan a partir de funciones continuas de RR procedentes de diferentes meta-análisis internacionales, la mayoría incluidos en una revisión reciente de Rehm et al (Corrao et al., 1999; Rehm et al., 2017; Samokhvalov et al., 2010) .

En bebedores actuales de $<100$ gramos de alcohol puro/ día, como $\mathrm{RR}_{\mathrm{i}}$ representativo de cada intervalo de exposición (cantidad media diaria de alcohol consumida) se utiliza la cifra $i$ correspondiente al punto medio de cada in- 
Tabla 2. Fracciones atribuibles poblacionales a alcohol para las causas seleccionadas, según sexo, período y edad. España, 2001-2017.

\begin{tabular}{|c|c|c|c|c|c|c|c|c|c|c|}
\hline & \multicolumn{10}{|c|}{ Hombres } \\
\hline & \multicolumn{5}{|c|}{ 2001-2009 } & \multicolumn{5}{|c|}{ 2010-2017 } \\
\hline & $15-24$ & $25-44$ & $45-64$ & $65-74$ & $\geq 75$ & $15-24$ & $25-44$ & $45-64$ & $65-74$ & $\geq 75$ \\
\hline \multicolumn{11}{|l|}{ Causa de muerte } \\
\hline 1. Tuberculosis & 0,427 & 0,563 & 0,628 & 0,576 & 0,511 & 0,341 & 0,440 & 0,573 & 0,601 & 0,474 \\
\hline 2. Infección respiratoria baja/neumonía & 0,111 & 0,157 & 0,188 & 0,171 & 0,159 & 0,084 & 0,119 & 0,167 & 0,185 & 0,155 \\
\hline 3. Cáncer de boca y faringe & 0,506 & 0,633 & 0,691 & 0,644 & 0,581 & 0,414 & 0,518 & 0,642 & 0,668 & 0,549 \\
\hline 4. Cáncer de esófago & 0,529 & 0,629 & 0,677 & 0,635 & 0,579 & 0,447 & 0,540 & 0,638 & 0,658 & 0,558 \\
\hline 5. Cáncer colorectal & 0,139 & 0,200 & 0,239 & 0,215 & 0,195 & 0,105 & 0,149 & 0,210 & 0,231 & 0,186 \\
\hline 6. Cáncer hepático & 0,369 & 0,586 & 0,670 & 0,607 & 0,528 & 0,286 & 0,394 & 0,592 & 0,631 & 0,451 \\
\hline 7. Cáncer de laringe & 0,483 & 0,497 & 0,501 & 0,493 & 0,460 & 0,240 & 0,318 & 0,418 & 0,443 & 0,348 \\
\hline 8. Cáncer de mama & - & - & - & - & - & - & - & - & - & - \\
\hline 9. Diabetes mellitus & $-0,056$ & $-0,042$ & $-0,021$ & $-0,018$ & $-0,008$ & $-0,060$ & $-0,058$ & $-0,033$ & $-0,016$ & $-0,011$ \\
\hline 10. Epilepsia & 0,290 & 0,396 & 0,455 & 0,409 & 0,357 & 0,225 & 0,302 & 0,407 & 0,434 & 0,335 \\
\hline 11. Cardiopatía hipertensiva & 0,210 & 0,292 & 0,342 & 0,305 & 0,269 & 0,161 & 0,221 & 0,303 & 0,327 & 0,254 \\
\hline 12. Cardiopatía isquémica & $-0,061$ & $-0,044$ & $-0,026$ & $-0,026$ & $-0,016$ & $-0,049$ & $-0,058$ & $-0,036$ & $-0,017$ & $-0,016$ \\
\hline 13. Fibrilación/aleteo auricular & 0,133 & 0,188 & 0,224 & 0,201 & 0,184 & 0,101 & 0,142 & 0,198 & 0,218 & 0,177 \\
\hline 14. Ictus isquémico & $-0,015$ & 0,040 & 0,090 & 0,076 & 0,074 & $-0,045$ & $-0,015$ & 0,056 & 0,087 & 0,065 \\
\hline 15. Ictus no isquémico & 0,157 & 0,222 & 0,263 & 0,234 & 0,208 & 0,119 & 0,166 & 0,232 & 0,252 & 0,198 \\
\hline 16. Cirrosis/hepatopatía crónica & 0,666 & 0,799 & 0,844 & 0,808 & 0,755 & 0,576 & 0,680 & 0,803 & 0,824 & 0,711 \\
\hline 17. Pancreatitis & 0,411 & 0,545 & 0,610 & 0,558 & 0,494 & 0,327 & 0,424 & 0,555 & 0,584 & 0,459 \\
\hline \multirow[t]{4}{*}{ 18. Causas externas } & 0,191 & 0,256 & 0,297 & 0,267 & 0,239 & 0,144 & 0,200 & 0,267 & 0,288 & 0,233 \\
\hline & \multicolumn{10}{|c|}{ Mujeres } \\
\hline & \multicolumn{5}{|c|}{ 2001-2009 } & \multicolumn{5}{|c|}{ 2010-2017 } \\
\hline & $15-24$ & $25-44$ & $45-64$ & $65-74$ & $\geq 75$ & $15-24$ & $25-44$ & $45-64$ & $65-74$ & $\geq 75$ \\
\hline \multicolumn{11}{|l|}{ Causa de muerte } \\
\hline 1. Tuberculosis & 0,245 & 0,251 & 0,246 & 0,197 & 0,144 & 0,220 & 0,222 & 0,257 & 0,219 & 0,158 \\
\hline 2. Infección respiratoria baja/neumonía & 0,063 & 0,070 & 0,068 & 0,057 & 0,051 & 0,059 & 0,066 & 0,073 & 0,066 & 0,056 \\
\hline 3. Cáncer de boca y faringe & 0,309 & 0,315 & 0,311 & 0,250 & 0,186 & 0,282 & 0,283 & 0,324 & 0,279 & 0,204 \\
\hline 4. Cáncer de esófago & 0,360 & 0,364 & 0,355 & 0,286 & 0,222 & 0,339 & 0,342 & 0,373 & 0,325 & 0,244 \\
\hline 5. Cáncer colorrectal & 0,077 & 0,085 & 0,083 & 0,069 & 0,059 & 0,072 & 0,079 & 0,088 & 0,079 & 0,065 \\
\hline 6. Cáncer hepático & 0,156 & 0,163 & 0,161 & 0,140 & 0,079 & 0,117 & 0,110 & 0,152 & 0,123 & 0,079 \\
\hline 7. Cáncer de laringe & 0,425 & 0,427 & 0,418 & 0,375 & 0,347 & 0,163 & 0,169 & 0,190 & 0,163 & 0,121 \\
\hline 8. Cáncer de mama & 0,132 & 0,138 & 0,135 & 0,107 & 0,085 & 0,121 & 0,127 & 0,144 & 0,124 & 0,094 \\
\hline 9. Diabetes mellitus & $-0,243$ & $-0,222$ & $-0,177$ & $-0,107$ & $-0,070$ & $-0,241$ & $-0,230$ & $-0,202$ & $-0,136$ & $-0,081$ \\
\hline 10. Epilepsia & 0,163 & 0,170 & 0,166 & 0,131 & 0,101 & 0,149 & 0,154 & 0,175 & 0,150 & 0,111 \\
\hline 12. Cardiopatía hipertensiva & 0,191 & 0,205 & 0,219 & 0,185 & 0,122 & 0,143 & 0,143 & 0,219 & 0,192 & 0,132 \\
\hline 12. Cardiopatía isquémica & $-0,052$ & $-0,032$ & $-0,014$ & 0,009 & 0,020 & $-0,059$ & $-0,042$ & $-0,019$ & 0,004 & 0,020 \\
\hline 13. Fibrilación/aleteo auricular & 0,075 & 0,082 & 0,080 & 0,066 & 0,057 & 0,070 & 0,077 & 0,086 & 0,076 & 0,062 \\
\hline 14. Ictus isquémico & $-0,154$ & $-0,136$ & $-0,095$ & $-0,045$ & $-0,044$ & $-0,186$ & $-0,183$ & $-0,122$ & $-0,081$ & $-0,054$ \\
\hline 15. Ictus no isquémico & 0,191 & 0,194 & 0,190 & 0,147 & 0,105 & 0,172 & 0,172 & 0,199 & 0,167 & 0,116 \\
\hline 16. Cirrosis/hepatopatía crónica & 0,430 & 0,434 & 0,429 & 0,363 & 0,249 & 0,380 & 0,370 & 0,433 & 0,373 & 0,264 \\
\hline 17. Pancreatitis & 0,234 & 0,240 & 0,236 & 0,189 & 0,139 & 0,211 & 0,213 & 0,246 & 0,210 & 0,152 \\
\hline 18. Causa externa & 0,107 & 0,114 & 0,112 & 0,089 & 0,074 & 0,100 & 0,107 & 0,120 & 0,105 & 0,082 \\
\hline
\end{tabular}

Nota. Las fracciones atribuibles poblacionales (FAP) para el grupo de causas de muertes directamente atribuibles a alcohol son siempre 1 y no se han incluido. Para calcular las FAP se han utilizado las prevalencias del consumo corregidas, elevando el consumo medio de cada participante en las encuestas hasta el $80 \%$ del consumo promedio per cápita estimado a partir de las estadísticas de venta. Para un cálculo más adecuado de las muertes atribuibles a alcohol para un determinado territorio o subgrupo a menudo se requieren FAP con 5 decimales, por lo que se recomienda solicitar el fichero a los autores.

tervalo $(10,30,45,55,70$ y 90$)$. En cambio, para bebedores actuales de $\geq 100$ gramos de alcohol/día se utiliza como $R_{i}$ representativo 130 gramos de alcohol puro/día, que es cantidad mediana de alcohol consumida por los bebedores de ese grupo incluidos en las muestras de las encuestas de salud poblacionales tanto en el período 2001-09 como en 2010-17. Además, en el caso de algunas causas de muerte se han utilizado RR específicos por sexo (diabetes mellitus, cardiopatía hipertensiva, cardiopatía isquémica, ictus isquémico, ictus no isquémico, cirrosis/hepatopatía crónica y pancreatitis). 
Tabla 3. Riesgos relativos de muerte por las causas seleccionadas, según sexo y nivel de exposición al alcohol. España, 2001-2017.

\begin{tabular}{|c|c|c|c|c|c|c|c|c|c|c|c|c|c|c|c|c|}
\hline & \multicolumn{8}{|c|}{ Hombres } & \multicolumn{8}{|c|}{ Mujeres } \\
\hline & \multicolumn{16}{|c|}{ Cantidad media de alcohol consumida (g alcohol puro/día) } \\
\hline & Exb $^{1}$. & 10 & 30 & 45 & 55 & 70 & 90 & 130 & Exb. & 10 & 30 & 45 & 55 & 70 & 90 & 130 \\
\hline 1. Tuberculosis & 1,38 & 1,20 & 1,71 & 2,24 & 2,69 & 3,52 & 5,04 & 10,34 & 1,38 & 1,20 & 1,71 & 2,24 & 2,69 & 3,52 & 5,04 & 10,34 \\
\hline 2. Infección respiratoria baja/neumonía & 1,38 & 1,05 & 1,15 & 1,24 & 1,30 & 1,40 & 1,54 & 1,86 & 1,38 & 1,05 & 1,15 & 1,24 & 1,30 & 1,40 & 1,54 & 1,86 \\
\hline 3. Cáncer de boca y faringe & 1,38 & 1,28 & 2,03 & 2,81 & 3,45 & 4,65 & 6,70 & 12,68 & 1,38 & 1,28 & 2,03 & 2,81 & 3,45 & 4,65 & 6,70 & 12,68 \\
\hline 4. Cáncer de esófago & 1,38 & 1,46 & 2,39 & 3,21 & 3,81 & 4,80 & 6,29 & 9,76 & 1,38 & 1,46 & 2,39 & 3,21 & 3,81 & 4,80 & 6,29 & 9,76 \\
\hline 5. Cáncer colorectal & 1,38 & 1,06 & 1,21 & 1,23 & 1,41 & 1,55 & 1,76 & 2,26 & 1,38 & 1,06 & 1,21 & 1,23 & 1,41 & 1,55 & 1,76 & 2,26 \\
\hline 6. Cáncer hepático & 1,38 & 1,01 & 1,16 & 1,40 & 1,66 & 2,29 & 3,94 & 17,55 & 1,38 & 1,01 & 1,16 & 1,40 & 1,66 & 2,29 & 3,94 & 17,55 \\
\hline 7. Cáncer de laringe & 1,38 & 1,16 & 1,52 & 1,85 & 2,10 & 2,52 & 3,17 & 4,77 & 1,38 & 1,16 & 1,52 & 1,85 & 2,10 & 2,52 & 3,17 & 4,77 \\
\hline 8. Cáncer de mama & - & - & - & - & - & - & - & - & 1,38 & 1,11 & 1,36 & 1,58 & 1,75 & 2,04 & 2,50 & 3,76 \\
\hline 9. Diabetes mellitus & 1,18 & 0,90 & 0,88 & 0,93 & 0,97 & 1,07 & 1,16 & 1,16 & 1,14 & 0,68 & 0,62 & 0,86 & 1,18 & 1,18 & 1,18 & 1,18 \\
\hline 11. Cardiopatía hipertensiva & 1,38 & 1,10 & 1,31 & 1,50 & 1,65 & 1,89 & 2,26 & 3,25 & 1,38 & 0,89 & 1,60 & 2,43 & 3,16 & 4,59 & 7,38 & 17,76 \\
\hline 12. Cardiopatía isquémica & 1,25 & 0,95 & 0,84 & 0,74 & 0,67 & 1,00 & 1,00 & 1,43 & 1,54 & 0,84 & 0,99 & 1,15 & 1,26 & 1,45 & 1,74 & 2,53 \\
\hline 13. Fibrilación/aleteo auricular & 1,38 & 1,06 & 1,19 & 1,30 & 1,37 & 1,50 & 1,68 & 2,11 & 1,38 & 1,06 & 1,19 & 1,30 & 1,37 & 1,50 & 1,68 & 2,11 \\
\hline 14. Ictus isquémico & 1,33 & 0,85 & 0,96 & 1,07 & 1,14 & 1,26 & 1,43 & 1,81 & 1,15 & 0,65 & 0,77 & 1,00 & 1,22 & 1,70 & 2,75 & 7,81 \\
\hline 15. Ictus no isquémico & 1,33 & 1,07 & 1,23 & 1,36 & 1,46 & 1,62 & 1,86 & 2,45 & 1,15 & 1,16 & 1,55 & 1,93 & 2,24 & 2,79 & 3,74 & 6,73 \\
\hline 16. Cirrosis/hepatopatía crónica & 1,31 & 1,33 & 2,32 & 3,53 & 4,67 & 7,10 & 12,42 & 37,95 & 6,50 & 2,82 & 5,97 & 8,90 & 11,20 & 15,24 & 21,93 & 40,87 \\
\hline 17. Pancreatitis & 1,38 & 1,19 & 1,68 & 2,18 & 2,60 & 3,37 & 4,76 & 9,53 & 1,38 & 0,77 & 0,66 & 1,53 & 3,01 & 4,80 & 10,26 & 10,26 \\
\hline 18. Causas externas & 1,38 & 1,09 & 1,30 & 1,48 & 1,62 & 1,85 & 2,21 & 2,42 & 1,38 & 1,09 & 1,30 & 1,48 & 1,62 & 1,85 & 2,21 & 2,42 \\
\hline
\end{tabular}

Exb': ex-bebedores.

Nota. Las referencias utilizadas para obtener los RR de cada causa de muerte son Corrao, Bagnardi, Zambon y Arico (1999); Samokhvalov, Irving y Rehm (2010) y Rehm et al. (2017), en el caso de los bebedores actuales. Para los exbebedores, las referencias son: Rehm et al. (2010a) y Stockwell et al. (2016).

En el caso de exbebedores no existen RR publicados para todas las causas de muerte seleccionadas. Para las causas específicas con información disponible, los RR se toman de un metanálisis de 2010 (Rehm et al., 2010a), y para aquellas sin información se aplica el RR de la mortalidad por todas las causas de un metanálisis de Stockwell et al, correspondiente al modelo conjunto y completamente ajustado, en concreto 1,38 (Stockwell et al., 2016).

\section{Prevalencias de exposición poblacional a alcohol}

En la fórmula de cálculo de las FAP interviene la prevalencia de exbebebores y las prevalencias anuales de distintas cantidades medias diarias de alcohol consumidas. Estas prevalencias por edad, sexo, y período se muestran en la Tabla 4.

A continuación se describe la fuente de datos y la forma de obtención de las mismas.

\section{Prevalencia de exbebedores}

Para el periodo 2010-2017 se estiman a partir de las cifras de la Encuesta Nacional de Salud (ENS) y la Encuesta Europea de Salud en España (EESE) correspondientes a ese período. Como los estudios que aportan RRs de mortalidad en exbebedores muy probablemente no incluyen a exbebedores infrecuentes o probadores, es recomendable corregir las prevalencias eliminando este subgrupo. Para efectuar esta corrección se utiliza la distribución de los exbebedores entre infrecuentes y regulares procedente de la Encuesta Nacional de Salud de Estados Unidos en el período 2011-14 (Villarroel et al., 2016), ya que no se encuentran datos en este sentido procedentes de fuentes españolas.

Para el período 2001-09 las cifras de prevalencia de exbebedores obtenidas de ENS y EESE son muy bajas en comparación con 2010-17 y con las obtenidas de otras fuentes para algunos subgrupos etarios, por lo que no se consideran fiables y se estiman a partir de las prevalencias corregidas de 2010-17, asumiendo un cambio relativo entre períodos similar al observado en la Encuesta Domiciliaria sobre Alcohol y Drogas en España (EDADES) (Delegación del Gobierno para el Plan Nacional sobre Drogas [DGPNSD], 2018).

\section{Prevalencias anuales de consumo medio diario de distintas cantidades de alcohol}

Se obtienen de los ficheros individuales de las ENS 2001, 2006, 2011 y 2017, y las EESE 2009 y 2014 (INE, 2019a; Ministerio de Sanidad, Consumo y Bienestar Social [MSCBS], 2019). Para ello, previamente se corrige por subestimación la cantidad media diaria de alcohol consumida autoinformada por cada participante durante el último año, ya que se sabe que los autoinformes de consumo de alcohol subestiman fuertemente el consumo real (Sordo et al., 2016). Las categorías de consumo consideradas para estratificar la 
Tabla 4. Prevalencia de consumo de alcohol corregida en la población de 15 años y más², según edad, sexo, cantidad media diaria de alcohol consumida y período (\%). España, 2001-2017.

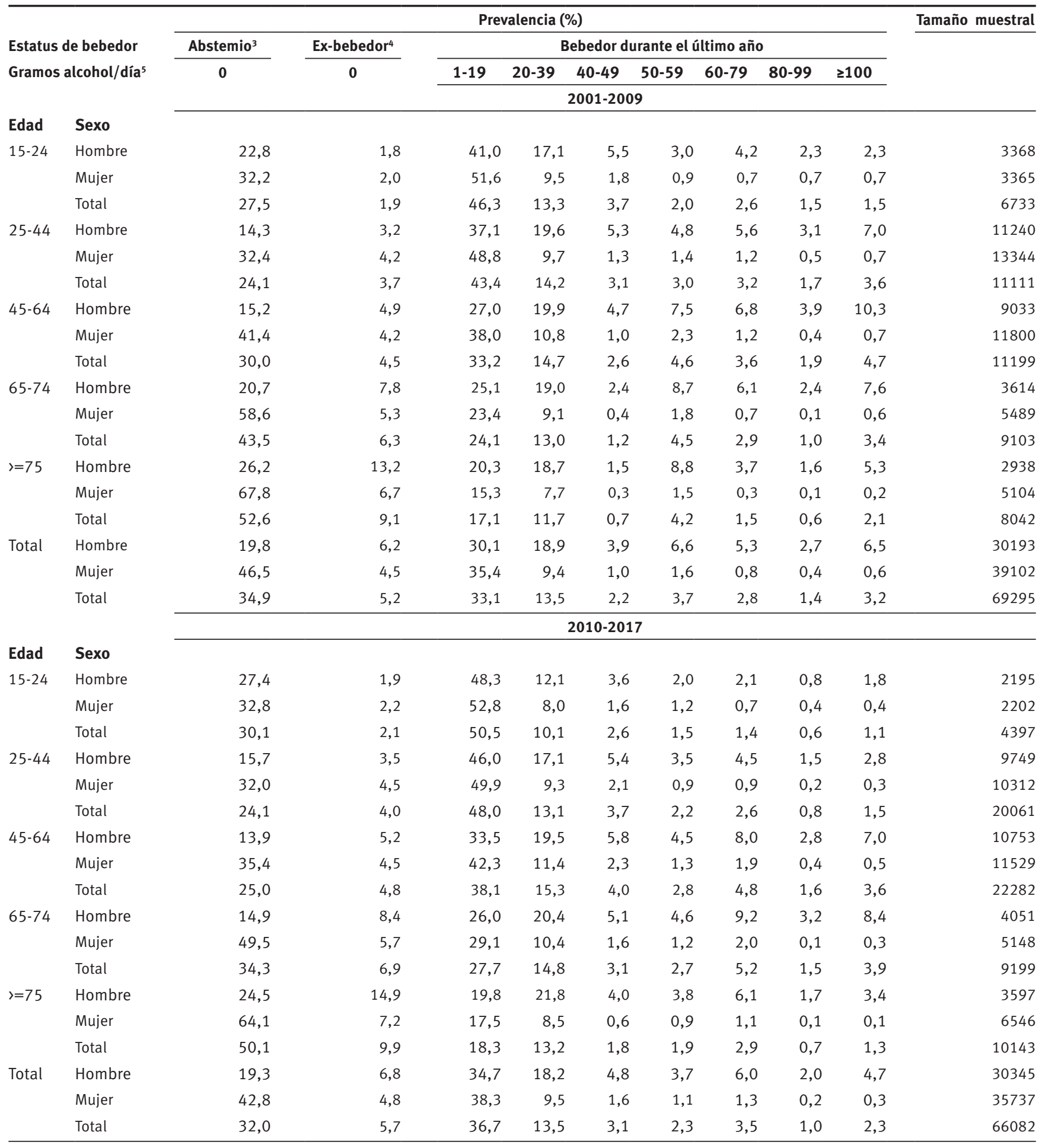

Nota. ${ }^{1}$ Prevalencia de consumo de alcohol corregida: Prevalencias obtenidas tras corregir la subestimación del consumo autoinformado en las encuestas poblacionales con respecto a las estadísticas de venta de consumo de alcohol. La corrección se efectuó aplicando un factor de elevación a la cantidad media diaria consumida por cada participante individual en la encuesta hasta el $80 \%$ de la cantidad media diaria per cápita estimada a partir de las estadísticas de venta. ${ }^{2}$ Población de 15 años y más: En realidad las prevalencias de consumo corresponden a la población de 16 años y más que es la población de referencia de las encuestas poblacionales incluidas en este trabajo. Sin embargo, las estimaciones de las estadísticas de venta procedentes de los organismos internacionales suelen aplicarse a la población de 15 años y más, por lo que las fracciones atribuibles a alcohol en la población y las estimaciones de la mortalidad atribuible se han aplicado a la población de 15 años y más. ${ }^{3}$ Abstemio: Persona que nunca en su vida ha consumido bebidas alcohólicas. ${ }^{4}$ Ex-bebedor: Persona que no ha consumido bebidas alcohólicas durante el último año y ha consumido estas bebidas menos de 12 veces cualquier año de su vida. La prevalencia fue estimada como se explica en el apartado Metodología. ${ }^{5}$ Alcohol consumido (g/día): Se refiere a la cantidad media de alcohol puro consumido diariamente en gramos.

prevalencia anual de consumo, en gramos de alcohol puro, corresponden a las 7 categorías $i$ mencionadas en el cálcu- lo de las FAPc. Las prevalencias para los años intermedios sin encuesta se estiman mediante interpolación lineal. 
A continuación, se detalla el algoritmo utilizado para corregir por subestimación. Para cada participante en la encuesta el consumo medio diario en un año dado en gramos de alcohol puro (Ac), se obtiene multiplicando el consumo medio diario autoinformado durante los 12 meses previos a la encuesta (Aa), por un factor de elevación (Fe). Este factor se calcula a su vez dividiendo la mejor estimación del consumo medio diario poblacional per cápita procedente de múltiples fuentes, principalmente estadísticas de ventas (Ar) entre Aa y multiplicando el resultado por el grado de corrección de la subestimación deseado (C).

$$
A c=\mathrm{Aa}\left[\frac{A r}{\mathrm{Aa}} C\right] \text {, donde }\left[\frac{A r}{\mathrm{Aa}} C\right]=\mathrm{Fe}
$$

De acuerdo con recomendaciones internacionales (Kehoe, Gmel, Shield, Gmel y Rehm, 2012; Rehm et al., 2010b; Stockwell et al., 2018), C se sitúa en 0,8; es decir, Aa se corrige hasta un $80 \%$ de Ar. La justificación de este proceder es algo prolija y se incluye en Apéndice Tabla 1. Las estimaciones de Aa, Ar y Fe por año calendario se incluyen en la Tabla 5. Como se puede apreciar en dicha tabla, el Fe ha aumentado en los años más recientes, lo que pone de manifiesto una dificultad creciente de las encuestas para capturar el consumo real y justifica aún más la necesidad de corregir el consumo autoinformado en las encuestas. Se calculó un Fe específico para cada año con encuesta y se aplicó a todos los participantes en la misma que habían consumido alcohol durante el último año, independientemente de su perfil sociodemográfico y sus patrones de consumo, porque solo se pudo estimar el Ar anual para el conjunto del país, sin posibilidad de estratificación según variables sociodemográficas.

La metodología para estimar Ar y Aa se ha publicado previamente (Sordo et al., 2016) y se resume a continuación.

\section{Estimación del consumo promedio de alcohol autoinformado (Aa)}

Las encuestas utilizadas para estimar $A a$ pueden considerarse representativas de la población de $\geq 15$ años no institucionalizada en España. Sus características pueden verse en Apéndice Tabla 2. La cantidad de alcohol consumida ( $A a$ ) en litros de alcohol puro (lap) por persona-año (pa) se estima siguiendo el enfoque clásico (Dawson, 2003) mediante el algoritmo: $A a=\sum_{i=1}^{k} \frac{D_{i} S D_{i} V_{i} C_{i}}{S S}$, donde el subíndice $i$ representa las distintas categorías de bebidas, como vino/ cava, cerveza/sidra, aperitivos/productos intermedios (bebidas con contenido alcohólico 1,2-22\% ABV distintas de las fermentadas, tipo vermut, jerez, oporto y vinos finos o amontillados) y licores/destilados (incluyendo combinados elaborados con licores), $\mathrm{D}_{\mathrm{i}}$ es el $\mathrm{n}^{\circ}$ anual de días o veces que se consume cada bebida, $\mathrm{SD}_{\mathrm{i}}$, el $\mathrm{n}^{\circ}$ de UBE consumidas cada día o cada vez, $\mathrm{V}_{\mathrm{i}}$ el volumen de cada UBE en litros, $\mathrm{C}_{\mathrm{i}}$ la proporción de alcohol sobre el volumen total

Tabla 5. Factores de elevación utilizados para corregir por subestimación el consumo anual de alcohol autoinformado. España, 2001-2017.

\begin{tabular}{|c|c|c|c|}
\hline & Consumo de alcohol registrado (Ar) ${ }^{1}$ & Consumo de alcohol autoinformado $(\mathrm{Aa})^{2}$ & Factor de elevación $(\mathrm{Fe})^{3}$ \\
\hline 2001 & 12,5 & 6,3 & 1,58 \\
\hline 2002 & 10,8 & & \\
\hline 2003 & 11,7 & & \\
\hline 2004 & 12,0 & & \\
\hline 2005 & 11,3 & & \\
\hline 2006 & 11,4 & 3,8 & 2,38 \\
\hline 2007 & 11,1 & & \\
\hline 2008 & 10,3 & & \\
\hline 2009 & 9,9 & 3,0 & 2,61 \\
\hline 2010 & 9,6 & & \\
\hline 2011 & 9,4 & 2,6 & 2,83 \\
\hline 2012 & 9,5 & & \\
\hline 2013 & 9,8 & & \\
\hline 2014 & 9,6 & 2,9 & 2,64 \\
\hline 2015 & 9,6 & & \\
\hline 2016 & 9,7 & & \\
\hline 2017 & 9,6 & 2,4 & 3,18 \\
\hline
\end{tabular}

Nota. ${ }^{1}$ Consumo de alcohol registrado (Ar): Consumo medio anual per cápita en litros de alcohol puro/año en residentes en España de 15 años y más estimado a partir de las estadísticas de venta. ${ }^{2}$ Consumo de alcohol autoinformado (Aa): Consumo medio anual per cápita en litros de alcohol puro/año en residentes en España de 15 años y más autoinformado en las encuestas poblacionales. ${ }^{3}$ Factor de elevación $(\mathrm{Fe})$ : $\mathrm{Fe}=(\mathrm{Ar} / \mathrm{Aa})^{\star}$ o,8. Es el factor multiplicador utilizado para corregir la subestimación del consumo de alcohol autoinformado en las estadísticas poblacionales con respecto a las estimaciones procedentes de las estadísticas de venta. Se hizo la corrección solo hasta el $80 \%$ del consumo estimado por las estadísticas de venta como se recomienda (Rehm et al., 2010a; Stockwell et al., 2018; Kehoe et al., 2012). Fue aplicado al consumo medio de cada individuo participante en las encuestas para posteriormente obtener las prevalencias poblacionales de consumo según cantidad media diaria consumida. 
de la bebida, y SS el tamaño muestral efectivo. Ante la gran escasez de datos empíricos (Rodríguez-Martos, Gual y Llopis Llácer, 1999), se asignan $\mathrm{V}_{\mathrm{i}}$ a las UBE de cada bebida en la línea alta de los volúmenes reflejados en algunas guías clínicas y de salud pública (Organización Médica Colegial y Ministerio de Sanidad y Consumo [OMC-MSC], 2006; Sociedad Española de Medicina de Familia y Comunitaria [SEMFYC], 2005): vino (125 ml), cerveza/sidra (250 ml), aperitivos $(100 \mathrm{ml})$, licores $(60 \mathrm{ml})$ y se aplican los $\mathrm{C}_{\mathrm{i}}$ para cada bebida propuestos por la Agencia Tributaria (5,5\%, $11,5 \%, 15 \%$ y $35 \%$, respectivamente) (Agencia Tributaria [AT], 2015; 2019). De esta manera, las cantidades de alcohol puro en gramos para la UBE de cada bebida resultan: 11,36 (vino), 10,86 (cerveza/sidra), 11,85 (aperitivos), y 16,59 (licores). Cuando se trata de una categoría poco concreta, por ejemplo, "bebidas locales", se asume que una UBE contiene $10 \mathrm{~g}$ de alcohol puro.

Los resultados de las encuestas se ponderaron para ajustar el desequilibrio de la muestra según sexo, grupo de edad, provincia, tamaño del hogar y tasa de respuesta.

\section{Estimación del consumo promedio de alcohol registrado $(\mathrm{Ar})$}

Los principales datos agregados para estimar el consumo medio registrado de alcohol per cápita (Ar) se refieren a ventas o suministros legales de bebidas con un contenido alcohólico en volumen $(\mathrm{ABV})>1,2 \%$, destinadas al consumo humano dentro de España. Estas cifras se corrigen para tener en cuenta el alcohol no registrado, el consumo/compras de viajeros internacionales, y las pérdidas de bebidas alcohólicas después de la venta. Los datos para estimar el alcohol consumido/comprado por visitantes extranjeros en España y visitantes españoles en el extranjero se obtienen del Instituto de Turismo de España (TURESPAÑA, 2019), INE (INE, 2019b), OMS (consumo de alcohol per cápita registrado por país) (WHO, 2020), Eurostat (Eurostat, 2019) y Banco Mundial (World-Bank, 2019) y bibliografía científica (Sordo et al., 2016). El resto de los datos necesarios para corregir las estadísticas de venta se obtienen de la bibliografía científica, incluyendo el consumo de bebidas de contrabando o fabricadas con alcohol destinado a otros usos, bebidas procedentes de la venta o producción informal o no controlada (por ejemplo autoconsumo), productos con un contenido alcohólico mayor de cero $\mathrm{y} \leq 1,2 \% \mathrm{ABV}$, pérdidas de bebidas alcohólicas (bebidas derramadas, estropeadas o desperdiciadas/no terminadas) y bebidas utilizadas para cocinar o para fines distintos al consumo humano directo (Boniface y Shelton, 2013; Landberg y Norstrom, 2011; Meier et al., 2013; Norstrom y Skog, 2001; Rehm et al., 2010b; Rehm et al., 2007; Trolldal, 2001; WHO, 2020). Las características de las principales fuentes rutinarias que aportan datos agregados útiles para estimar el consumo per cápita de alcohol en España se incluyen en Apéndice Tabla 3.
La cantidad de alcohol se expresa en litros de alcohol puro por persona-año (lap/pa), usando como denominador la población residente en España cada año. Para obtener el consumo real de alcohol, se siguió un proceso de múltiples etapas a partir de la disponibilidad de alcohol para el consumo de la AT. La AT calcula la disponibilidad agregando al alcohol procedente de ventas legales de bebidas sujetas a impuestos especiales sobre el alcohol (cerveza, licores y aperitivos) el alcohol procedente de compras de vino autoinformadas al Panel de Consumo Alimentario. Esta disponibilidad no incluye sidra, y se ha señalado que las compras de vino autoinformadas están subestimadas respecto a las ventas (como ocurre en el caso de la cerveza, donde la subestimación llega a ser de 45\%). Por lo tanto, se construye un indicador de disponibilidad de fuentes múltiples reemplazando el componente de vino en la disponibilidad de AT por el suministro de vino de Eurostat y agregando el suministro de sidra de FAO. Finalmente, el consumo medio real per cápita se obtiene agregando el alcohol procedente de las bebidas alcohólicas consumidas/compradas en el extranjero por residentes en España, y el resto del alcohol consumido y no registrado, y restando las pérdidas de alcohol y el alcohol procedente de bebidas consumidas/compradas en España por visitantes extranjeros. Los algoritmos concretos de cálculo pueden consultarse en un trabajo previo (Sordo et al., 2016).

\section{Indicadores de mortalidad atribuible a alcohol}

Los principales indicadores utilizados para expresar los resultados de las estimaciones de la mortalidad atribuible a alcohol en España son: número absoluto de MAA y varios indicadores estandarizados por edad como tasa de MAA (TMAA), y contribución proporcional del alcohol al riesgo de mortalidad general (CAMG), contribución proporcional al riesgo total de mortalidad atribuible a alcohol de distintas causas de muerte (CMAAC), y del consumo de alto riesgo (CMAAR).

La TMAA es un indicador del riesgo absoluto o probabilidad de morir a causa del consumo de alcohol en un subgrupo de población dado. Las tasas poblacionales se expresan por 100.000 personas-año (pa) y se estandarizan usando el método directo con los pesos de la estructura etaria de la Población Estándar Europea de 2013 (Eurostat, 2013). Las contribuciones proporcionales estandarizadas se expresan como porcentaje y son el resultado de dividir la TMAA entre la tasa de mortalidad por todas las causas (CAMG), la TMAA específica por cada causa entre la TMAA por todas las causas (CMAAC), y la TMAA en bebedores actuales de alto riesgo entre la suma de las TMAA en bebedores de alto riesgo y de medio-bajo riesgo (CMAAR).

La mayor parte de los indicadores se calculan por sexo, grupo de edad (15-34, 35-54, 55-74 años y >=75 años), comunidad autónoma, periodo calendario (2001-09 Y 2010- 
17) y tipo de bebedor (exbebedores regulares, bebedores de medio-bajo riesgo y bebedores de alto riesgo).

Las poblaciones por grupo de edad, sexo, comunidad autónoma y año-calendario para el cálculo de los indicadores se obtuvieron de las Cifras de Población del Instituto Nacional de Estadística (INE, 2019c). Para comparar el riesgo de MAA entre grupos (por ejemplo, hombres y mujeres) o períodos (2001-09 y 2010-17) se utilizaron la razón y la diferencia de tasas estandarizadas por edad, transformando la razón de tasas en porcentaje de cambio para las comparaciones temporales.

\section{Análisis de sensibilidad}

Se realizan varios análisis de sensibilidad para observar cómo afecta el cambio de algunas opciones metodológicas a la estimación del $\mathrm{n}^{\circ}$ total de MAA en 2017 (Apéndice Tabla 4). Entre ellos, introducir prevalencias sin corrección por subestimación del consumo, no considerar en los cálculos las MAA que se producen en exbebedores, adoptar una definición de exbebedor más amplia que incluye tanto "exbebedores regulares" como "exbebedores infrecuentes", operar con las prevalencias de consumo de alcohol del período 2001-09 en vez de las del período 20102017 , lo que equivale a introducir un período de latencia de aproximadamente 12 años con respecto a 2017, y usar como FAPc para estimar las MAA por accidente de tráfico la proporción de conductores y peatones fallecidos en estos accidentes con una alcoholemia superior a 0,8 gramos / litro durante 2015-17, calculadas con datos extraídos de las Memorias anuales del procedentes del Instituto Nacional de Toxicología y Ciencias Forenses durante 2001-17 (1870 conductores y 489 peatones) (Instituto Nacional de Toxicología y Ciencias Forenses [INTCF], 2019).

\section{Discusión de la metodología}

En este artículo se establece una metodología detallada para estimar la mortalidad atribuible a alcohol en España, siguiendo en la medida de lo posible las recomendaciones y los procederes internacionales más comunes y actualizados en este campo (IHME, 2020; Rehm et al., 2017; Rehm et al., 2009; Sherk et al., 2017; WHO, 2000, 2018b, 2020). Su aplicación al período 2001-2017 ha permitido obtener resultados según sexo, edad, período calendario, comunidad autónoma, causa de muerte y tipo de bebedor, que son bastante consistentes con otros indicadores de problemas de alcohol. En el futuro permitirá a su vez obtener resultados comparativos según otros subgrupos de interés, como los definidos por el nivel de estudios o la posición socioeconómica a partir de las cohortes de mortalidad de los censos de 2001 y 2011 de la Plataforma KharonXXI de Estudios Nacionales Longitudinales de Mortalidad que se está constituyendo en el marco de CIBERESP.
Las dos principales fortalezas de la metodología son la obtención de FAPc a partir de datos empíricos de exposición poblacional al alcohol procedentes casi en su totalidad de fuentes españolas, y que los datos individuales sobre consumo promedio de alcohol autoinformados en las encuestas se corrigen por subestimación en relación a otras fuentes de mayor validez como las estadísticas de venta de bebidas alcohólicas, siguiendo un proceso muy cuidadoso (Sordo et al., 2016). Además, la metodología tiene otras fortalezas, como la inclusión de las MAA ligadas a exbebedores. Finalmente, las FAPc obtenidas son bastante robustas porque la distribución de la exposición poblacional al alcohol se basa en el análisis conjunto de encuestas con un tamaño muestral importante ( $\mathrm{n}=66082$ en 2010-2017 y 69295 en 2001-2009). Ello posibilita la obtención de FAPc para los 20 subgrupos poblacionales mencionados arriba y segmentar la FAPc de cada causa de muerte por tipo de bebedor (exbebedor, bebedor de medio-bajo riesgo y bebedor de alto riesgo).

A pesar de sus fortalezas y de que se ha realizado un gran esfuerzo para recoger todos los datos relevantes disponibles, se trata sin duda de un primer desarrollo, que habrá que revisar y mejorar en el futuro. En este sentido, existen bastantes opciones metodológicas del modelo propuesto que pueden discutirse.

Se opta por un enfoque de causa específica frente al de todas las causas, porque permite un mejor control de la confusión, así como estimar el peso relativo de distintas causas o grupos de causa de muerte en la mortalidad total atribuible a alcohol, lo que tiene interés para la práctica clínica y de salud pública (Corrao, Rubbiati, Zambon y Arico, 2002). No se trata solo de conocer el número de MAA, sino también las causas concretas para diseñar intervenciones de prevención y tratamiento más ajustadas.

Más allá de este enfoque general, se opta por estrategias metodológicas conservadoras para evitar sobreestimar el $\mathrm{n}^{\mathrm{o}}$ de MAA, lo que seguramente implica que las cifras obtenidas subestiman la mortalidad atribuible a alcohol en España. Entre estas estrategias destacan: 1) Solo se incluyen aquellas causas de muerte en que hay evidencia clara de su relación con el consumo de alcohol y estimaciones válidas de los RR (Tabla 1). Esto deja fuera un grupo amplio de enfermedades probablemente relacionadas con el alcohol. 2) En los cálculos de la cantidad de alcohol consumida, se aplica al vino una gradación alcohólica de 11,5\% ABV, que es probablemente baja en el contexto español actual (Alston, Lapsley, Soleas y Tumber, 2013). 3) Se aplica una definición de exbebedor restrictiva, incluyendo solo a la fracción de estas personas (exbebedores regulares), que posiblemente se adapta mejor al perfil de exbebedores a que se refieren los RR obtenidos en los estudios epidemiológicos. Esta opción, que probablemente no se ha adoptado en otros estudios (Rehm et al., 2010a; Stockwell et al., 2016), rebaja considerablemente las estimaciones del $n^{\circ}$ de 
MAA porque los exbebedores tienen RR cercanos a los bebedores de alto riesgo (Rehm et al., 2010a; Stockwell et al., 2016), al haber dejado muchos el consumo de alcohol por problemas derivados de su consumo. En cualquier caso, hay que tener en cuenta que los datos para efectuar la corrección proceden de Estados Unidos, donde la situación epidemiológica del consumo de alcohol puede no ser equivalente a la española. Por ejemplo, en España ha descendido claramente el consumo de alcohol durante el siglo XXI, cosa que no ha sucedido en Estados Unidos (Breslow, Castle, Chen y Graubard, 2017; Centers for Disease Control and Prevention [CDC], 2020). El efecto de incluir en los cálculos sólo a los exbebedores regulares o al conjunto de los exbebedores puede apreciarse en Apéndice Tabla 4. 4) No se ha tratado el consumo intensivo episódico de manera específica debido a la falta de información al respecto; se considera, entonces, que este patrón de consumo ya se tiene en cuenta en el algoritmo utilizado para obtener las FAPs de forma general. No obstante, en Apéndice Tabla 4 pueden observarse los cambios en la estimación derivados de considerar el efecto de este patrón de consumo. 5) Los $R R_{i}$ de algunas enfermedades están probablemente subestimados, porque se calculan en comparación con personas abstemias, un grupo que en algunos estudios puede incluir también a exbebedores regulares (Rehm et al., 2017; Rehm, Shield, Roerecke y Gmel, 2016; Stockwell et al., 2016). Se ha realizado, no obstante, un análisis de sensibilidad para 2017, considerando un período de latencia de aproximadamente 12 años y la estimación del número de MAA es sólo 1,03 veces mayor que la considerada válida en este estudio (Apéndice Tabla 4).

Existen, por otra parte, algunas limitaciones generales que afectan a las estimaciones de la mortalidad atribuible a alcohol en todo el mundo. Entre ellas hay que mencionar la escasez y baja calidad de las estimaciones de los $R R_{i}$ publicados para ciertas causas de muerte, grupos de edad, sexo y consumo promedio diario. Así los $R R_{i}$ para algunos problemas de salud agudos, por ejemplo, accidentes de tráfico, pueden variar ampliamente por edad, por lo que la aplicación del mismo $R R_{i}$ a todas las edades podría provocar una subestimación de estas muertes en personas jóvenes y una sobreestimación en personas mayores (Jones, Bellis, Dedman, Sumnal y Tocke, 2008; Rehm, Patra y Popova, 2006). Las estimaciones de MAAs para los grupos de edad más viejos y algunas causas de muerte, como las enfermedades circulatorias, son particularmente dependientes de las opciones metodológicas referidas a exposición a alcohol o $\mathrm{RR}_{\mathrm{i}}$ elegidas para realizarlas (Marmet et al., 2016; Sherk, Thomas, Churchill y Stockwell, 2020; Trias-Llimós, Martikainen, Mäkelä y Janssen, 2018). Además, los $R R_{i}$ utilizados reflejan seguramente la fuerza de la asociación entre el consumo promedio y la mortalidad por causa en países y momentos con unos patrones de consumo diferentes a los prevalentes en España en el período de estudio. Finalmente, señalar al respecto que los RR, al relacionar el consumo con la incidencia, normalmente no separan entre morbilidad y mortalidad, por lo que en ocasiones se hace referencia a incidencia de enfermedad y no necesariamente de mortalidad. No obstante, no existe una manera alternativa de obtener estos $R R_{i}$. Otra limitación se relaciona con la práctica de corregir por subestimación de manera homogénea en el conjunto de participantes en las encuestas poblacionales. Si como es previsible, existen diferencias en el grado de subestimación según factores sociodemográficos o nivel de consumo promedio diario de alcohol esto podría sesgar las estimaciones del $n^{\circ}$ total de MAA, así como su distribución según factores sociodemográficos, causa de muerte y tipo de bebedor.

Como se ha indicado, esta metodología debería servir de base para poner en marcha en España un indicador rutinario de mortalidad atribuible a alcohol que contribuya a monitorizarla, comenzando por un análisis de las limitaciones de los sistemas generales de información sanitaria existentes. En este sentido, se ha de mejorar y mantener la consistencia temporal de las medidas de consumo de alcohol en las encuestas de salud poblacionales, así como las estadísticas de ventas de bebidas alcohólicas. Los cambios frecuentes en el contenido y formato de las preguntas sobre consumo de alcohol en los cuestionarios de la Encuesta Nacional de Salud durante el período 2001-2017, o la incertidumbre asociada a los datos de ventas de bebidas alcohólicas no sujetas a impuesto especial (vino y sidra) publicados por la AT o las instituciones internacionales, han dificultado enormemente la estimación de la exposición poblacional al alcohol durante ese período. La adopción de instrumentos internacionales no ha mejorado necesariamente la situación, porque a menudo no se adaptan demasiado a los patrones de consumo y tipos de bebidas predominantes en España. Igualmente, sería deseable conocer los cambios en las prácticas de certificación y codificación de las causas de muerte en España, especialmente en lo relativo a enfermedades circulatorias, para poder interpretar mejor los hallazgos sobre la evolución de la mortalidad atribuible a alcohol según causa.

\section{Reconocimientos}

Este artículo es un producto de trabajos realizados en el marco de un proyecto de investigación financiado por la Delegación del Gobierno para el Plan Nacional sobre Drogas (DGPNSD) [N Exp: 2015I040] y del apoyo con recursos humanos del Instituto de Salud Carlos III (Contrato ISCIII-PFIS, N ${ }^{\circ}$ Expediente: ENPY-397/18-PFIS). Se agradece también la colaboración de Cristina Ortiz del ISCIII, que realizó el análisis del consumo de alcohol de alguna de las encuestas poblacionales utilizadas para las estimaciones, y del Grupo Alcohol de la Sociedad Española de Epidemiología, que apoyó el desarrollo de este trabajo. 


\section{Conflicto de intereses}

Los autores manifiestan que no tienen ningún conflicto de intereses relacionado con los aspectos tratados en este artículo.

\section{Referencias}

Agencia Europea de Medicamentos. (2010). Guideline on the development of medicinal products for the treatment of alcohol dependence. Recuperado de https:/ / www.ema.europa. $\mathrm{eu} / \mathrm{en} /$ documents/scientific-guideline/guideline-development-medicinal-products-treatment-alcohol-dependence_en.pdf.

Agencia Tributaria. (2015). Impuestos especiales. Recuperado de http://www.agenciatributaria.es/AEAT.internet/Inicio/Aduanas_e_Impuestos_Especiales/Impuestos_Especiales/Impuestos_Especiales.shtml.

Agencia Tributaria. (2019). Impuestos sobre el alcohol y las bebidas alcohólicas, 2015. Recuperado de https://www. agenciatributaria.es/AEAT.internet/Inicio/La_Agencia_Tributaria/Memorias_y_estadisticas_tributarias/ Estadisticas/Estadisticas_por_impuesto/Impuestos_especiales/Impuestos_especiales.shtml.

Alston, J. M. F., Lapsley, J.T., Soleas, G. y Tumber K.P. (2013). The high and rising alcohol content of wine. En. E. P. Giraud-Héraud, M.C. (Ed.), Wine Economics. London: Palgrave Macmillan.

Boniface, S. y Shelton, N. (2013). How is alcohol consumption affected if we account for under-reporting? A hypothetical scenario. European Journal of Public Health, 23, 1076-1081. doi:10.1093/eurpub/ckt016.

Breslow, R. A., Castle, I. P., Chen, C. M. y Graubard, B. I. (2017). Trends in alcohol consumption among older americans: national health interview surveys, 1997 to 2014. Alcoholism: Clinical and Experimental Research, 41, 976-986. doi:10.1111/acer.13365.

Centers for Disease Control and Prevention. (2020). Alcohol use. Recuperado de https://www.cdc.gov/nchs/fastats/alcohol.htm.

Connor, J., Kydd, R., Rehm, J. y Shield, K. (2013). Alcohol-attributable burden of disease and injury in New Zealand: 2004 and 2007. Research report commissioned by the Health Promotion Agency. Recuperado de https://www.hpa.org. $\mathrm{nz} /$ sites/default/files/Attributable $\% 20$ fractions $\% 20$ Final.pdf.

Corrao, G., Bagnardi, V., Zambon, A. y Arico, S. (1999). Exploring the dose-response relationship between alcohol consumption and the risk of several alcohol-related conditions: a meta-analysis. Addiction, 94, 1551-1573. doi:10.1046/j.1360-0443.1999.9410155111.x.

Corrao, G., Rubbiati, L., Zambon, A. y Arico, S. (2002). Alcohol-attributable and alcohol-preventable mortality in
Italy. A balance in 1983 and 1996. European Journal of Public Health, 12, 214-223. doi:10.1093/eurpub/12.3.214.

Dawson, D. A. (2003). Methodological issues in measuring alcohol use. Alcohol Research E Health, 27, 18-29.

Delegación del Gobierno para el Plan Nacional sobre Drogas. (2018). EDADES 2017. Encuesta sobre alcohol y otras drogas en España (EDADES), 1995-2017. Recuperado de http://www.pnsd.mscbs.gob.es/profesionales/ sistemasInformacion/sistemaInformacion/pdf/EDADES_2017_Informe.pdf.

Eurostat. (2013). Revision of the European Standard Population. Report of Eurostat's task force. Recuperado de https:/ / ec.europa.eu/eurostat/documents/3859598/5926869/KSRA-13-028-EN.PDF/e713fa79-1add-44e8-b23d-5e8fa09b3f8f.

Eurostat. (2019). Purchasing power parities (PPPs), price level indices and real expenditures for ESA 2010 aggregates. Recuperado de https://ec.europa.eu/eurostat/web/products-datasets/-/prc_ppp_ind\&lang=en.

Fierro, I., Ochoa, R., Yánez, J. L., Valderrama, J. C. y Álvarez, F. J. (2008). Mortalidad y mortalidad prematura relacionadas con el consumo de alcohol en España entre 1999 y 2004. Medicina Clínica, 131, 10-13. doi:10.1157/13123036.

Global Burden of Disease. (2018a). Alcohol use and burden for 195 countries and territories, 1990-2016: a systematic analysis for the Global Burden of Disease Study 2016. Lancet, 392, 1015-1035. doi:10.1016/S01406736(18)31310-2.

Global Burden of Disease. (2018b). Global, regional, and national comparative risk assessment of 84 behavioural, environmental and occupational, and metabolic risks or clusters of risks for 195 countries and territories, 19902017: A systematic analysis for the Global Burden of Disease Study 2017. Lancet, 392, 1923-1994. doi:10.1016/ S0140-6736(18)32225-6.

Gmel, G., Shield, K. D. y Rehm, J. (2011). Developing a method to derive alcohol-attributable fractions for HIV/ AIDS mortality based on alcohol's impact on adherence to antiretroviral medication. Population Health Metrics 9, 5. doi:10.1186/1478-7954-9-5.

Institute for Health Metrics and Evaluation. (2020). GBD Results Tool. Recuperado de http://ghdx.healthdata. org/gbd-results-tool.

Instituto Nacional de Estadística. (2019a). Encuesta Nacional de Salud. Microdatos. Recuperado de https://www. ine.es/dyngs/INEbase/es/operacion.htm?c=Estadistica_C\&cid=1254736176783\&menu=resultados\&sec$\mathrm{c}=1254736195295$ \&idp=1254735573175.

Instituto Nacional de Estadística. (2019b). INEbase. Servicios. Hosteleria y turismo. Recuperado de https://www. ine.es/dyngs/INEbase/es/categoria.htm?c=Estadistica_P\&cid=1254735576863. 
Instituto Nacional de Estadística. (2019c). INEbase. Demografía y población. Cifras de población y censos demográficos. Cifras de población. Recuperado de https://www. ine.es/dyngs/INEbase/es/operacion.htm?c=Estadistica_C\&cid=1254736176951\&menu=ultiDatos\&i$\mathrm{d} p=1254735572981$.

Instituto Nacional de Estadística. (2020). INEbase Sociedad. Salud. Estadística de defunciones según la causa de muerte. Resultados. 2020. Recuperado de https://www. ine.es/dyngs/INEbase/es/operacion.htm?c=Estadistica_C\&cid=1254736176780\&menu=resultados\&i$\mathrm{dp}=1254735573175 \#$ !tabs-1254736194710.

Instituto Nacional de Toxicología y Ciencias Forenses. (2019). Memorias. Víctimas mortales en accidentes de tráfico. Recuperado de https://www.mjusticia.gob.es/cs/Satellite/Portal/va/ministerio/organismos-ministerio-justi$\mathrm{cia} /$ instituto-nacional/memorias.

Jones, L. y Bellis, M. A. (2013). Updating England-Specific Alcohol-Attributable Fractions. Recuperado de http:/ /www. cph.org.uk/wp-content/uploads/2014/03/24892-ALCOHOL-FRACTIONS-REPORT-A4-singles-24.3.14.pdf.

Jones, L., Bellis, M. A., Dedman, D., Sumnal, H. y Tocke, C. (2008). Alcohol-attributable fractions for England. Alcohol-attributable mortality and hospital admissions. Recuperado de http:/ / www.nwph.net/nwpho/publications/alcoholattributablefractions.pdf.

Kehoe, T., Gmel, G., Shield, K. D., Gmel, G. y Rehm, J. (2012). Determining the best population-level alcohol consumption model and its impact on estimates of alcohol-attributable harms. Population Health Metrics, 10, 6. doi:10.1186/1478-7954-10-6.

Landberg, J. y Norstrom, T. (2011). Alcohol and homicide in Russia and the United States: a comparative analysis. Journal of Studies on Alcohol and Drugs, 72, 723-730. doi:10.15288/jsad.2011.72.723.

Mann, K., Aubin, H. J. y Witkiewitz, K. (2017). Reduced drinking in alcohol dependence treatment, what is the evidence? European Addiction Research, 23, 219-230. doi:10.1159/000481348.

Marmet, S., Rehm, J. y Gmel, G. (2016). The importance of age groups in estimates of alcohol-attributable mortality: Impact on trends in Switzerland between 1997 and 2011. Addiction, 111, 255-262. doi:10.1111/add.13164.

Marmet, S., Rehm, J., Gmel, G., Frick, H. y Gmel, G. (2014). Alcohol-attributable mortality in Switzerland in 2011-age-specific causes of death and impact of heavy versus non-heavy drinking. Swiss Medical Weekly, 144, w13947. doi:10.4414/smw.2014.13947.

Meier, P. S., Meng, Y., Holmes, J., Baumberg, B., Purshouse, R., Hill-McManus, D. y Brennan, A. (2013). Adjusting for unrecorded consumption in survey and per capita sales data: quantification of impact on gender- and age-specific alcohol-attributable fractions for oral and pharyngeal cancers in Great Britain. Alcohol and Alcoholism, 48, 241-249. doi:10.1093/alcalc/agt001.

Ministerio de Sanidad, Consumo y Bienestar Social. (2019). Banco de datos. Recuperado de https://www.mscbs.gob. es/estadEstudios/estadisticas/bancoDatos.htm.

Norstrom, T. y Skog, O. J. (2001). Alcohol and mortality: Methodological and analytical issues in aggregate analyses. Addiction, 96 (Suppl 1), S5-S17. doi:10.1080/09652140020021143.

Organización Médica Colegial y Ministerio de Sanidad y Consumo. (2006). Guía de buena práctica clínica en uso y abuso de alcohol. Recuperado de http://www.msssi.gob. es/alcoholJovenes/docs/guiaBPCusoabusoAlcohol07. pdf.

Rehm, J., Baliunas, D., Borges, G. L., Graham, K., Irving, H., Kehoe, T., ...Taylor, B. (2010a). The relation between different dimensions of alcohol consumption and burden of disease: an overview. Addiction, 105, 817-843. doi:10.1111/j.1360-0443.2010.02899.x.

Rehm, J., Gmel, G. E., Sr., Gmel, G., Hasan, O. S. M., Imtiaz, S., Popova, S., ...Shuper, P. A. (2017). The relationship between different dimensions of alcohol use and the burden of disease-an update. Addiction, 112, 968-1001. doi:10.1111/add.13757.

Rehm, J., Kehoe, T., Gmel, G., Stinson, F., Grant, B. y Gmel, G. (2010b). Statistical modeling of volume of alcohol exposure for epidemiological studies of population health: the US example. Population Health Metrics, 8, 3. doi:10.1186/1478-7954-8-3.

Rehm, J., Klotsche, J. y Patra, J. (2007). Comparative quantification of alcohol exposure as risk factor for global burden of disease. International Journal of Methods in Psychiatric Research, 16, 66-76. doi:10.1002/mpr.204.

Rehm, J., Mathers, C., Popova, S., Thavorncharoensap, M., Teerawattananon, Y. y Patra, J. (2009). Global burden of disease and injury and economic cost attributable to alcohol use and alcohol-use disorders. Lancet, 373, 22232233. doi:10.1016/S0140-6736(09)60746-7.

Rehm, J., Patra, J. y Popova, S. (2006). Alcohol-attributable mortality and potential years of life lost in Canada 2001: implications for prevention and policy. Addiction, 101, 373-384. doi:10.1111/j.1360-0443.2005.01338.x.

Rehm, J., Rehm, M. X., Shield, K. D., Gmel, G. y Gual, A. (2013). Alcohol consumption, alcohol dependence and related harms in Spain, and the effect of treatment-based interventions on alcohol dependence. Adicciones, 25, 11-18.

Rehm, J., Shield, K. D., Rehm, M. X., Gmel, G. y Frick, U. (2012). Alcohol consumption, alcohol dependence and attributable burden of disease in Europe. Potential gains from effective interventions for alcohol dependence. Recuperado de http:/ / amphoraproject.net/w2box/data/AMPHORA\%20Reports/CAMH_Alcohol_Report_Europe_2012.pdf. 
Rehm, J., Shield, K. D., Roerecke, M. y Gmel, G. (2016). Modelling the impact of alcohol consumption on cardiovascular disease mortality for comparative risk assessments: an overview. BMC. Public Health, 16, 363. doi:10.1186/s12889-016-3026-9.

Rey, G., Boniol, M. y Jougla, E. (2010). Estimating the number of alcohol-attributable deaths: methodological issues and illustration with French data for 2006. Addiction, 105, 1018-1029. doi:10.1111/j.1360-0443.2010.02910.x.

Rodríguez-Martos, D. A., Gual, S. A. y Llopis Llácer, J. J. (1999). The "standard drink unit" as a simplified record of alcoholic drink consumption and its measurement in Spain. Medicina Clínica, 112, 446-450.

Roerecke, M. y Rehm, J. (2013). Alcohol use disorders and mortality: A systematic review and meta-analysis. Addiction, 108, 1562-1578. doi:10.1111/add.12231.

Samokhvalov, A. V., Irving, H. M. y Rehm, J. (2010). Alcohol consumption as a risk factor for atrial fibrillation: a systematic review and meta-analysis. European Journal of Cardiovascular Prevention E Rehabilitation, 17, 706-712. doi:10.1097/HJR.0b013e32833a1947.

Sherk, A., Stockwell, T., Rehm, J., Dorocicz, J. y Shield, K. D. (2017). The International Model of Alcohol Harms and Policies (InterMAHP). Version 1.0: December 2017. Recuperado de www.intermahp.cisur.ca.

Sherk, A., Thomas, G., Churchill, S. y Stockwell, T. (2020). Does drinking within low-risk guidelines prevent harm? Implications for high-income countries using the international model of alcohol harms and policies. Journal of Studies on Alcohol and Drugs, 81, 352-361.

Sociedad Española de Medicina de Familia y Comunitaria. (2005). Guía rápida para abordar el abuso de alcohol. Recuperado de https://www.semfyc.es/pfw_files/cma/ Informacion/modulo/documentos/abuso-alcohol.pdf.

Sordo, L., Barrio, G., Bravo, M. J., Villalbí, J. R., Espelt, A., Neira, M. y Regidor, E. (2016). Estimating average alcohol consumption in the population using multiple sources: The case of Spain. Population Health Metrics, 14, 21. doi:10.1186/s12963-016-0090-4.

Stockwell, T., Zhao, J., Panwar, S., Roemer, A., Naimi, T. y Chikritzhs, T. (2016). Do "moderate" drinkers have reduced mortality risk? A systematic review and meta-analysis of alcohol consumption and all-cause mortality. Journal of Studies on Alcohol and Drugs, 77, 185-198. doi:10.15288/jsad.2016.77.185.

Stockwell, T., Zhao, J., Sherk, A., Rehm, J., Shield, K. y Naimi, T. (2018). Underestimation of alcohol consumption in cohort studies and implications for alcohol's contribution to the global burden of disease. Addiction, 113, 2245-2249. doi:10.1111/add.14392.

Trias-Llimós, S., Martikainen, P., Mäkelä, P. y Janssen, F. (2018). Comparison of differents approaches for estimating age-specific alcohol-attributable mortality: The cases of France and Finland. PLoS One, 13: e0194478. doi:10.1371.journal.pone.0194478.

Trolldal, B. (2001). Alcohol sales figures in 15 European countries: Corrected for consumption abroad and tax-free purchases. Nordic Studies on Alcohol, 18, 71-81. doi:10.1177/145507250101801S02.

TURESPAÑA. (2019). Estadísticas turísticas. Recuperado de http:/ / estadisticas.tourspain.es/es-ES/turismobase/Paginas/default.aspx.

Villarroel, M. A., Clarke, T. C. y Schoenborn, C. A. (2016). Tables of adult health behaviors, alcohol use: National Health Interview Survey, 2011-2014., from National Center for Health Statistics. Center for Disease Control and Prevention (CDC). Recuperado de https://ftp.cdc.gov/pub/Health_Statistics/NCHs/NHIS/SHS/2011-2014_AHB_Table_ALC-1.pdf.

World-Bank. (2019). International Comparison Program. ICP 2011. Recuperado de https://datacatalog.worldbank. org/dataset/international-comparison-program-2011.

World Health Organization. (2000). International guide for monitoring alcohol consumption and related harm. WHO/ MSD/MSB/00.4. Recuperado de http://whqlibdoc.who. int/hq/2000/who_msd_msb_00.4.pdf.

World Health Organization. (2009). Global health risks. Mortality and burden of disease attributable to selected major risks. WA 105. Recuperado de http://www.who.int/healthinfo/global_burden_disease/GlobalHealthRisks_report_ full.pdf.

World Health Organization. (2018a). Methods and data sources for global burden of disease estimates, 2000-2016. Recuperado de https://www.who.int/healthinfo/global_burden_disease/GlobalDALY_method_2000_2016.pdf.

World Health Organization. (2018b). Global status report on alcohol and health. Recuperado de https://www.who.int/ substance_abuse/publications/alcohol/en/.

World Health Organization. (2020). Global Information System on Alcohol and Health (GISAH). Recuperado de http://apps.who.int/gho/data/node.gisah.GISAH?showonly=GISAH. 